efficacy and common tolerability challenges, provided that the studies used for these calculations are similar enough. Number needed to harm $(\mathrm{NNH})$ values may be even more helpful when distinguishing among treatments that are relatively otherwise similar. $^{2}$ The NNH can be for overall tolerability (discontinuation because of an adverse effect) or the occurrence of specific adverse effects of concern for individual patients being treated (such as sedation, weight gain or akathisia). Moreover, ratios of $\mathrm{NNH}$ to NNT can provide overall estimates of the risk-benefit trade-offs involved. Finally, we suggest that all of the above concepts are straightforward enough for average clinicians to calculate and understand.,

1 Roose SP, Rutherford BR, Wall MM, Thase ME. Practising evidence-based medicine in an era of high placebo response: number needed to treat reconsidered. Br J Psychiatry 2016; 208: 416-20.

2 Ketter TA, Miller S, Dell'Osso B, Calabrese JR, Frye MA, Citrome L. Balancing benefits and harms of treatments for acute bipolar depression. I Affect Disord 2014; 169: S24-33.

3 Citrome L, Ketter TA. When does a difference make a difference? Interpretation of number needed to treat, number needed to harm, and likelihood to be helped or harmed. Int J Clin Pract 2013; 67: 407-11.

4 Citrome L, Ketter TA. Teaching the philosophy and tools of evidence-based medicine: misunderstandings and solutions. Int J Clin Pract 2009; 63: 353-9.

Leslie Citrome, Clinical Professor of Psychiatry and Behavioral Sciences, New York Medical College, Valhalla, New York USA. Email: citrome@cnsconsultant.com; Terence A. Ketter, Professor of Psychiatry and Behavioral Sciences

doi: 10.1192/bjp.209.3.262b

Authors' reply: Drs Citrome and Ketter appear to appreciate the concern we raised about the limitations of applying the NNT from placebo-controlled studies to the clinical situation (where there is no placebo control condition). However, in their letter they maintain that, 'Indirect comparisons of effect sizes among different medication choices can be quite helpful in ranking interventions for both efficacy and common tolerability challenges, provided that the studies used for these calculations are similar enough'. We do not disagree; in fact, we quoted Garcia in our paper: 'to directly compare NNTs one needs to ensure that [...] the control or comparisons groups to which the treated group was compared were equivalent.'

Our point in the paper was that insufficient attention is typically paid to the question of whether control conditions are 'similar enough', and we believe this point still holds. Although it is not clear from their letter to what type of situation Drs Citrome and Ketter refer, one is likely on firmest ground when comparing NNTs and NNHs for antidepressant medications calculated from placebo-controlled trials of similar methodology and quality. However, even in this optimal case, it has been established that placebo response can vary significantly from trial to trial, and thus the control conditions for two studies may in fact be less similar than one might suppose. ${ }^{2}$

Perhaps it would be less problematic to compare the NNTs and $\mathrm{NNHs}$ calculated from a comparator trial of two or more antidepressants, because of course in this case there is no issue about the similarity of the studies. The problem is that, to our knowledge, there has not been a consistent finding that one antidepressant has therapeutic superiority or greater tolerability compared with another. One must be careful not to use the NNT and NNH from a single study when that finding has not been replicated, especially since comparator studies are primarily industry-sponsored.

Beyond the specific case of comparing two antidepressant medications, the points made by Citrome and Keller are not relevant to the fundamental thesis of our paper that NNTs calculated from placebo-controlled trials do not inform the clinician's choice whether to prescribe or not prescribe. Additionally, our further point still stands that NNHs and NNTs cannot be applied without significant confounding to decisions of whether to prescribe medications or psychotherapy, since the control conditions for these treatments are usually radically different.

1 Garcia AM. What does "work" mean? Reopening the debate about clinical significance. Clin Psychol Sci Pract 2010; 17: 48-51.

2 Walsh BT, Seidman SN, Sysko R, Gould M. Placebo response in studies of major depression: variable, substantial, and growing. JAMA 2002; 287: 1840-7.

Steven P. Roose, MD, Columbia University College of Physicians and Surgeons, New York State Psychiatric Institute, New York, USA. Email: spr2@cumc.columbia.edu; Bret R. Rutherford, MD

doi: 10.1192/bjp.209.3.263

\section{Challenges in developing feasible and cost-effective therapies for use in LMICs}

Chowdhary et al conducted the research reported in their paper ${ }^{1}$ under the aegis of PREMIUM (a Program for Mental Health Interventions for Under-Resourced Health systems) in India. They state the overall aim of this programme in their introduction: 'to investigate a systematic, reproducible method for developing psychological treatments that incorporate global evidence, are contextually appropriate and can be delivered by non-specialist health workers'. In this paper, the authors set out to develop an intervention to be delivered by lay health workers, with the intention of addressing the treatment gap for mental health. The elaborate methodology they adopted to develop this intervention requires a highly skilled research team such as their own. There are simpler and more economical methods for cultural adaptation of evidence-based therapies ${ }^{2,3}$ that have been tested in similar cultures and well described. We are not clear about the rationale for their use of a complex and expensive methodology, given the aim of a 'reproducible method for developing psychological treatments'. The authors started with a pool of techniques that were considered to be useful. These techniques were mostly based on cognitive-behavioural therapy (CBT). However, based on expert advice, they adapted the manual Behavioral Activation for Depression: A Clinician's Guide. A massive evaluation found this intervention to be unfeasible. Therefore, they further adapted the intervention and tested it in a pilot study. The title of their paper does not reflect the fact that this was an adaptation of an existing intervention and not the development of a new intervention. They used a complex, time-consuming and resource-intensive process that is highly unlikely to be repeatable in low- and middle-income countries (LMICs).

We have adapted CBT for the local population in Pakistan and for the ethnic minority population in England. ${ }^{2,3}$ These methods of adaptation have been described in detail and have been tested for depression ${ }^{4}$ and schizophrenia, ${ }^{3,5}$ and in a guided self-help format for depression. ${ }^{6}$ The methodology evolved over the years, resulting in the development of semi-structured interviews that can be conducted by students and easily analysed using a framework analysis method. ${ }^{5}$ This low-cost methodology is being used in China and the Middle East to adapt CBT. We hope the authors find this work useful in their future attempts to adapt therapy.

The issue of cost becomes even more important in the delivery of therapy. In our two-pronged approach, therapy in secondary care was delivered by psychology graduates (with a typical monthly salary of \$200) and by carers using a culturally adapted CBT-based self-help manual developed locally. No financial help was provided to the carers. We believe it is not just the development or adaptation of an intervention that is important; it should also be deliverable by existing mechanisms. This leads to our second concern: how practical it is to create a new workforce of lay therapists in a low-income country? This lack of understanding of the ground realities has possibly resulted in minimal change 